\title{
Fallstudie Kanton Zürich: «E-Umzug - Online Umziehen»
}

Lukas Steudler und Elke Brucker-Kley

5.1 Kontext und Ausgangssituation - 70

5.2 Motivation und Zielsetzung - 72

5.3 Umsetzung und Wirkung - 73

$5.4 \quad$ Fazit -78

Literatur - 81 
In der Schweiz ziehen jährlich rund 700.000 Haushalte um (ISB 2015a), 200.000 davon im Kanton Zürich. Damit verbunden ist die Verpflichtung, sich bei der Gemeinde um- bzw. abzumelden und bei der Zuzugsgemeinde anzumelden. eUmzugZH ermöglicht Einwohnern und Einwohnerinnen im Kanton Zürich seit April 2016, diese Meldungen unabhängig von Schalteröffnungszeiten via Internet zu erledigen. Eingebettet in ein nationales Vorhaben eUmzugCH soll dies zukünftig auch schweizweit möglich sein. Die Erfahrungen bei der Umsetzung von eUmzugZH sowie die Herausforderungen und Lösungsansätze, einen durchgängigen Meldeprozess in einem föderalen System zu realisieren, sind Gegenstand der vorliegenden Fallstudie.

\subsection{Kontext und Ausgangssituation}

Digitalisierung ist ein wesentliches Element einer modernen, bürgernahen und effizienten Verwaltung. Einwohner und Einwohnerinnen haben zunehmend das Bedürfnis, Behördengänge nicht mehr persönlich und zeitaufwendig am Schalter, sondern jederzeit online von zuhause oder unterwegs zu erledigen. Aber auch für die Verwaltung bietet ein mehrstufiger und mit Medienbrüchen behafteter Prozess wie die Administration eines Um-, Zu- oder Wegzugs die Chance, durch den Einsatz zeitgemässer Informationstechnologien Ineffizienzen zu beseitigen.

॥ E-Government ist selbstverständlich: transparente, wirtschaftliche und medienbruchfreie elektronische Behördenleistungen für Bevölkerung, Wirtschaft und Verwaltung (Leitbild der eGovernment-Strategie Schweiz (ISB 2016)).

Vor diesem Hintergrund wurde 2012 auf Ebene Bund ein priorisiertes E-GovernmentVorhaben A1.12 mit dem Titel eUmzugCH definiert, mit dem Ziel «schweizweit den elektronischen, papierfreien Umzug für schweizerische und ausländische Staatsangehörige in der Schweiz zu ermöglichen» (VSED 2012, S. 4). Der Bund reagierte damit zum einen auf die sich verändernde Mobilität der Bevölkerung und zum anderen auf die zunehmende Komplexität eines stark dezentralisierten Meldewesens, das an die rund 2600 Städte und Gemeinden in der Schweiz delegiert ist (VSED 2012). Diese setzen für die Einwohnerkontrolle unterschiedliche IT-Lösungen von Gemeindesoftwareanbietern ein, die vereinzelt bereits einen Umzug als Online-Service ermöglichen. Der Nutzen ist jedoch auf Umzüge innerhalb einer Gemeinde oder zwischen Gemeinden, die das gleiche System nutzen, begrenzt. Um einen Umzug unabhängig von der eingesetzten Gemeindesoftware flächendeckend zwischen verschiedenen Gemeinden eines Kantons oder kantonsübergreifend anbieten zu können, strebt das Vorhaben A1.12 eine schweizweite Harmonisierung und Standardisierung der Meldeprozesse bei Adressänderungen sowie Weg- und Zuzug an, ohne dabei in die Kompetenzen von Gemeinden und Kantonen einzugreifen.

\section{- Das elektronische Meldewesen in der Schweiz}

Wesentliche rechtliche und technische Grundlagen für ein elektronisches Meldewesen wurden in der Schweiz bereits mit dem Projekt «Registerharmonisierung» und dem Registerharmonisierungsgesetz ${ }^{1}$ geschaffen. Eine Schlüsselrolle spielt in diesem Kontext die Datenübermittlungsplattform des Bundesamts für Statistik «sedex» (= secure data exchange), die seit 2008 den gesetzlich geregelten, sicheren und standardisierten Datenaustausch zwischen den angeschlossenen amtlichen Registern von Bund, Kantonen und Gemeinden sicherstellt (BfS, o.J.). Auch

\footnotetext{
${ }^{1}$ RHG, Registerharmonisierungsgesetz, vom 23.06.2006, SR 431.02.
} 
die Einwohnerregister der Städte und Gemeinden wurden sukzessive an sedex angeschlossen. Eine weitere Grundlage bilden die Standards für das elektronische Meldewesen des Vereins eCH, der im Rahmen der E-Government-Strategie Schweiz Daten- und Schnittstellenstandards entwickelt (eCH, o.J.) $)^{2}$. So ermöglicht der Standard eCH-0093 bereits seit 2014 bei Zu- und Wegzug den Datenaustausch zwischen Einwohnerregistern von Gemeinden unabhängig von Hersteller, Kanton oder Sprachgrenzen.

\section{- Kanton Zürich als Vorreiter}

Standards und eine nationale Datenaustauschplattform allein bewirken jedoch noch nicht, dass der elektronische Umzug online auch für die Bevölkerung gemeindeübergreifend und schweizweit Realität wird. Damit die im Hintergrund zwischen den Gemeinden stattfindenden Meldeprozesse aus Sicht eines umziehenden Einwohners einfach in einem Schritt, durchgängig und nachvollziehbar ablaufen, braucht es eine Plattform, die eine einheitliche Benutzeroberfläche und die Prozessintegration sicherstellt. Eine solche Infrastruktur in Einzelprojekten auf Ebene der Gemeinden zu realisieren, ergibt wenig Sinn, würde zu enormen Redundanzen in der Entwicklung führen und wertvolle Synergieeffekte verschenken. Eine Alternative stellen Lösungen dar, die eine durchgängige Umzugsmeldung zwischen Gemeinden, die das gleiche Einwohnerkontroll(EK)-System einsetzen, ermöglichen. Allerdings lässt sich auf diesem Weg ein flächendeckendes Angebot nur bedingt realisieren, da beispielsweise allein im Kanton Zürich EK-Systeme von sechs verschiedenen Anbietern im Einsatz sind, deren Lösungen standardkonform miteinander integriert werden müssten, um Umzüge zwischen allen Gemeinden abzudecken. Kantonale Lösungen reduzieren diese Komplexität, indem sie den Meldeprozess unabhängig von den EK-Systemen oder EK-Verbünden in den Gemeinden allen Einwohnern des Kantons anbieten und die Integration mit den verschiedenen EK-Systemen und externen Informationsquellen (z. B. Gebäude- und Wohnregister) zentral lösen. Kantonale Lösungen erscheinen somit wirksame Hebel zu sein, um die Umsetzung des priorisierten E-Government-Vorhabens A1.12 konzertiert und effizient voranzutreiben. Der Kanton Zürich hat ab 2014 die Vorreiterrolle in der Schweiz für die Umsetzung einer solchen kantonalen Lösung übernommen und hat eUmzugZH gemeinsam mit den teilnehmenden Gemeinden seit April 2016 erfolgreich eingeführt. Das Zusammenspiel zwischen Kanton und Gemeinden wurde dabei von der Zusammenarbeitsorganisation egovpartner.zh.ch (o.J.), die bei der Staatskanzlei des Kantons Zürich angesiedelt ist, sichergestellt. egovpartner.zh.ch wurde von Kanton und Gemeinden auf der Basis einer Kooperationsvereinbarung gegründet (eGovernmentZH 2012), mit der Zielsetzung E-Government-Projekte effizient durchzuführen und Synergiepotenziale auszunutzen. Der Fokus von egovpartner.zh.ch liegt auf dem Aufbau moderner Online-Angebote mit den Gemeinden des Kantons Zürich, um Bevölkerung und Unternehmen Amtsgeschäfte zunehmend auch in digitaler Form ermöglichen zu können. Die vorliegende Fallstudie beschreibt die Umsetzung und Wirkung des Projekts eUmzugZH aus der Perspektive von egovpartner.zh. ch.

\footnotetext{
${ }^{2}$ Eine aktuelle Aufstellung der eCH-Standards in der Fachgruppe Meldewesen findet sich auf der eCH Website: http://www.ech.ch/vechweb/page?p=categorylist \&site=/documents/alle/nach\%20Fachgruppe.
} 


\subsection{Motivation und Zielsetzung}

Ziel von eUmzugZH ist es, der Bevölkerung in den Gemeinden des Kantons Zürich Umzug, Weg- und Zuzug als Online-Service flächendeckend anzubieten. Darüber hinaus fliessen die Ergebnisse in ein technologieneutrales Referenzmodell ein, das die erarbeiteten Prozesse, Schnittstellen, Standards und Empfehlungen für eUmzugs-Projekte in anderen Kantonen wiederverwendbar macht. Die Möglichkeit eines kantonsübergreifenden eUmzugCH gemäss Zielsetzung des priorisierten E-Government-Vorhabens A1.12 soll so schrittweise realisiert werden.

Nach Aussagen des Schweizerischen Verbands der Einwohnerdienste ist die Möglichkeit, die Meldevorgänge im Rahmen eines Umzuges elektronisch durchzuführen, die am meisten nachgefragte E-Government-Dienstleistung (VSED 2012, S. 4). Sowohl von Seiten der Bevölkerung als auch von Seiten der Gemeinden besteht offensichtlich ein grosses Interesse, diesen zeitaufwendigen und - im Falle eines Umzuges zwischen zwei Gemeinden - zweistufigen Prozess einfacher und schneller über das Internet abwickeln zu können. Die Aufwände auf beiden Seiten reduzieren zu können und das Meldewesen bei einem Umzug somit effizienter und kundenorientierter gestalten zu können, sind die wesentlichen Motivationen für den elektronischen Umzug. In diesem Sinne wird auch ein Beitrag zur Umsetzung der E-GovernmentStrategie Schweiz geleistet, die Effizienz und Dienstleistungsorientierung als zwei von vier strategischen Zielen formuliert.

\section{E-Government-Strategie Schweiz}

2007 hat der Bundesrat die erste E-Government-Strategie Schweiz verabschiedet, die ein gemeinsames Leitbild und Ziele für die gemeinsame Umsetzung durch Bund, Kantone und Gemeinden formuliert. Die Strategie wurde von der Geschäftsstelle E-Government Schweiz weiterentwickelt und Ende 2015 in aktualisierter Form vom Bundesrat, der Konferenz der Kantonsregierungen sowie den Vorständen des Städte- und des Gemeindeverbandes unterzeichnet. Darin sind unter anderem die vier strategischen Ziele festgehalten (ISB 2016):

1. Dienstleistungsorientierung: Die elektronischen Behördenleistungen sind einfach nutzbar, transparent und sicher.

2. Nutzen und Effizienz: E-Government schafft für Bevölkerung, Wirtschaft und Behörden einen Mehrwert und reduziert bei allen Beteiligten den Aufwand bei der Abwicklung von Behördengeschäften.

3. Innovation und Standortförderung: E-Government nutzt Innovationen und fördert damit die Attraktivität des Wirtschaftsstandortes und Lebensraumes Schweiz.

4. Nachhaltigkeit: Die Mehrfachnutzung von Lösungen wird gefördert. Bund und Kantone stellen die Nachhaltigkeit von E-Government-Diensten sicher, indem sie Voraussetzungen für deren Organisation, Finanzierung und den Betrieb schaffen.

Ein wesentliches Element der E-Government-Strategie ist die koordinierte Umsetzung durch Bund, Kantone und Gemeinden (ISB 2016). Gemeinsam formulierte Ziele, Grundsätze, Vorgehen und Instrumente sollen ermöglichen, dass Synergien genutzt und die Durchgängigkeit gewährleistet wird. Bund und Kantone stehen dabei in der Pflicht, die «Nachhaltigkeit von E-Government-Diensten» sicherzustellen, indem sie unter anderem die «Mehrfachnutzung von Lösungen» fördern und die «Voraussetzungen für deren Organisation, Finanzierung 
und Betrieb» schaffen. eUmzugCH mit dem darin eingebetteten eUmzugZH zeigen, wie in diesem Sinne ein Vorhaben auf Ebene Bund priorisiert und auf Ebene Kanton gemeinsam mit den Gemeinden umgesetzt wird. Die Ziele von eUmzugZH und eUmzugCH sind daher zusammenhängend zu betrachten:

- Umzug sowie Weg- und Zuzug in Gemeinden des Kantons Zürich können gemäss Zielsetzung des priorisierten Vorhabens A1.12 elektronisch abgewickelt werden. Die Ausbreitung im Kanton Zürich erfolgt flächendeckend und koordiniert (egovpartner.zh.ch). Im Anschluss an eine Pilot- und Einführungsphase soll der elektronische Umzug als OnlineService bis Ende 2016 allen Zürcher Gemeinden zur Verfügung stehen.

- Ziel ist es, aus der Perspektive der umziehenden Einwohner und Einwohnerinnen einen durchgängigen, einfach verständlichen und transparenten Meldeprozess anzubieten, der gleichzeitig die Aufwände auf Seiten der Gemeinden reduziert.

- Zielgruppen sind volljährige Personen mit Schweizer Bürgerrecht sowie Personen mit ausländischer Nationalität mit Ausnahme bestimmter Aufenthaltskategorien im Asylbereich (Ausweis $\mathrm{N}$ und $\mathrm{S}$ )

- Ausgehend von den Erkenntnissen aus der Umsetzung im Kanton Zürich wird ein lösungsneutrales Referenzmodell geschaffen, das für die Umsetzung in weiteren Kantonen wiederverwendet werden kann.

Für die Umsetzung im Kanton Zürich gelten die folgenden Grundsätze:

- Das Meldewesen im Kanton Zürich ist eine Hoheitsaufgabe der Gemeinden. Die Gemeinde und deren Einwohnerkontrollsysteme bleiben bestehen und fachlich führend. Der Kanton stellt lediglich eine Plattform für den Dateneingang (Benutzeroberfläche) und die Prozessintegration zur Verfügung.

- Die Plattform muss eine intuitive Benutzung gewährleisten. Eine Supportorganisation für Anfragen aus der Bevölkerung wird nicht bereitgestellt. Bei Problemen verbleibt der Weg zum Schalter.

- Die Meldung eines Umzugs, Wegzugs oder Zuzugs muss weiterhin auch am Schalter möglich sein, um alle Bevölkerungsgruppen bedienen zu können.

- Da der elektronische Umzug nicht von Beginn an flächendeckend im Kanton Zürich und noch nicht über die Kantonsgrenzen hinaus durchgängig angeboten werden kann, sollen verschiedene Szenarien bzw. Einstiegspunkte in den elektronischen Umzugsprozess unterstützt werden. Bietet die Zuzugs- bzw. Wegzugsgemeinde noch keinen elektronischen Umzug an, kann auch nur ein Teil des Prozesses online erfolgen (z. B. der Wegzug), während der andere Teil (z. B. der Zuzug) am Schalter stattfindet.

I) Die Gemeinden und die kantonale Verwaltung verkehren untereinander elektronisch und tauschen Daten aus. Bevölkerung und Unternehmen des Kantons Zürich können die wichtigsten Amtsgeschäfte und Anliegen online, ohne Medienbruch und jeweils über möglichst eine Anlaufstelle (Single Point of Contact) tätigen. Einer der am meisten genannten Services ist der elektronische Umzug (Vision von eUmzugZH (egovpartner.zh.ch)).

\subsection{Umsetzung und Wirkung}

Um Einwohnern und Einwohnerinnen im Kanton Zürich bei einem Umzug einen durchgängigen Meldeprozess über die Gemeindegrenzen hinaus online anbieten zu können, mussten technische, rechtliche und organisatorische Voraussetzungen geschaffen werden. Mit eUmzugZH 

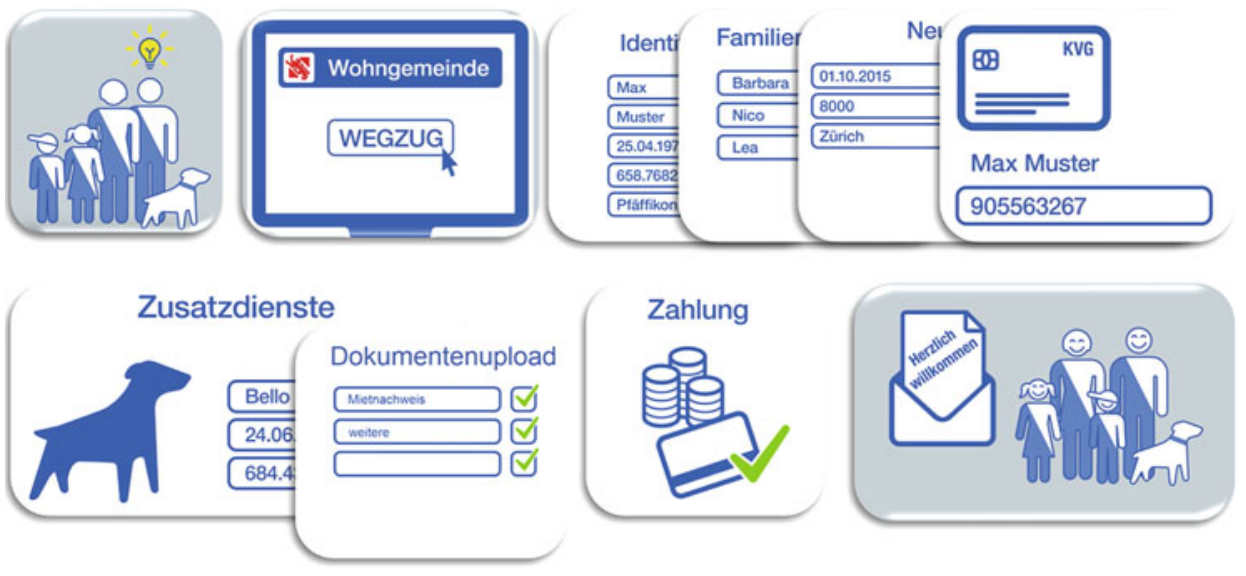

- Abb. 5.1 eUmzugZH aus der Perspektive des Nutzers

ist dies gelungen. Nach nur zwei Monaten und noch vor Abschluss der Einführungsphase nutzt die Bevölkerung den Dienst bereits rege und mit positiver Resonanz. Der flächendeckenden Ausdehnung im Kanton Zürich steht nichts mehr im Wege und mit dem geschaffenen Referenzmodell wird die Umsetzung in weiteren Kantonen beschleunigt.

Seit der Einführung von eUmzugZH im April 2016 konnte die Reichweite bereits von initial acht Pilotgemeinden auf rund 49 der 168 Zürcher Gemeinden ausgedehnt werden (Stand Juli 2016). In dieser Zeit wurden bereits rund 1300 Umzüge online gemeldet. • Abb. 5.1 skizziert die Schritte, die die Nutzer dabei online durchlaufen.

1. Über die Website der aktuellen Wohngemeinde (= Wegzugsgemeinde) gelangt der Nutzer auf die Website von eUmzugZH, auf der er den Wegzug meldet. Bietet die Wegzugsgemeinde noch keinen elektronischen Umzug an, sondern nur die Zuzugsgemeinde, kann der Nutzer auch direkt den Zuzug online melden und den Wegzug auf konventionelle Weise erledigen. Auch das umgekehrte Szenario ist möglich, d. h. die Meldung des Wegzugs online und die Meldung des Zuzugs am Schalter. Auf diese Weise ist eine Online-Umzugsmeldung während der Übergangsfrist bis zum flächendeckenden Angebot zumindest in Teilen möglich. Der elektronische Austausch der Daten zwischen den Gemeinden kann im Hintergrund dennoch elektronisch stattfinden und über die Benutzerführung kann die Vollständigkeit der Unterlagen für den Behördengang sichergestellt werden. Zu Beginn des Dialogs erfasst der Nutzer eine E-Mail-Adresse, an die nach erfolgreicher Prüfung des Wegund Zuzugs eine Bestätigung versandt wird.

2. Der Nutzer identifiziert sich mit Namen, Geburtsdatum, Wohnort und AHV-Nummer. Durch eine synchrone Abfrage über sedex (eCH-0194) im Einwohnerregister der Wegzugsgemeinde ${ }^{3}$ wird mit diesen Angaben die Identität geprüft. Gleichzeitig werden Personen, die im selben Haushalt leben, an den Benutzer zurückgemeldet, damit sie gegebenenfalls in die Umzugsmeldung mit eingeschlossen werden können. Auf eine Registrierung, d. h. die Erstellung eines Benutzerkontos bei der Gemeinde, wird bei eUmzugZH bewusst verzichtet, um die Einstiegshürden möglichst gering zu halten und einem Einwohner nicht die

\footnotetext{
${ }^{3}$ In Kantonen mit einer zentralen Einwohnerdatenplattform kann die Identifikation zentral erfolgen. So muss nicht jede Gemeinde an die synchrone Abfrage angebunden werden. Im Kanton Zürich läuft das Projekt «KEP» mit dem Ziel eine kantonale Einwohnerdatenplattform bis Ende 2018 einzuführen.
} 
Einrichtung eines Benutzerkontos in einer Gemeinde zuzumuten, die er ohnehin verlässt. Zudem wird das Schadenspotenzial bei einer Umzugsmeldung als gering eingestuft, so dass auf aufwendigere Formen der Authentifizierung verzichtet wird.

3. Im nächsten Schritt erfasst der Nutzer die neue Adresse in der Zuzugsgemeinde. Diese Daten werden mit dem eidgenössischen Gebäude- und Wohnungsregister abgeglichen.

4. Da die Zuzugsgemeinde für die Prüfung der Krankenversicherungspflicht (KVG-Obligatorium) zuständig ist, muss der Nutzer die Krankenversicherungsnummern von allen Familienangehörigen erfassen. Diese werden über eine Online-Abfrage beim VeKa-Center (Versicherungskarten-Center) überprüft.

5. Anschliessend können noch Zusatzdienste wie beispielsweise die Meldung eines Hundes genutzt werden. Auch Dokumente wie z. B. ein Mietnachweis, die von den Gemeinden unter Umständen verlangt werden, können hochgeladen werden.

6. Abschliessend erfolgt die Zahlung der Gebühren über Kreditkarte oder Postcard online.

7. Die Daten werden an die Wegzugsgemeinde übermittelt, die den Vorgang prüft und zur Weiterverarbeitung an die Zuzugsgemeinde übermittelt. Der Nutzer erhält abschliessend die Meldebestätigung per E-Mail und damit die Quittung, dass der Weg- und Zuzug erfolgreich gemeldet wurde. Im Verlauf des Prozesses hat der Nutzer jederzeit die Möglichkeit, den Status der Meldung online abzufragen.

\section{- Kantonale Federführung in der Umsetzung}

Einen solch durchgängigen E-Government-Prozess im föderalen System der Schweiz zu realisieren ist kein einfaches Unterfangen. Im Fall von eUmzugZH galt es, vor dem Hintergrund eines nationalen E-Government-Vorhabens, die Kompetenzen von Kanton und Gemeinden anzuerkennen und in das Lösungs- und Betriebskonzept einzubeziehen. Auch wenn die Führung der Einwohnerregister eine hoheitliche Aufgabe der Gemeinden ist, übernahm der Kanton im Rahmen von eUmzugZH die Federführung und schaffte in Zusammenarbeit mit den Gemeinden und den Anbietern der Einwohnerkontrollsysteme die technischen, rechtlichen und organisatorischen Voraussetzungen für einen durchgängig digitalisierten Umzugsmeldeprozess.

\section{- Technische Voraussetzungen}

Implementiert wurde die Lösung mit externen Realisierungspartnern auf der kantonalen Transaktionsplattform ZHservices. ZHservices dient dem sicheren elektronischen Amtsverkehr und stellt bereits andere E-Government-Dienste für die Bevölkerung und Unternehmen des Kantons Zürich zur Verfügung, wie beispielsweise die Online-Steuererklärung oder die Online-Abwicklung der Quellensteuer. Für eUmzugZH stellt ZHservices zum einen die Benutzeroberfläche für die Interaktion mit den meldepflichtigen Einwohnern und Einwohnerinnen zur Verfügung. Zum anderen wird die Prozessintegration sichergestellt, d.h. die kontrollierte Ausführung des Meldeprozesses von der Identifikation bis zum Abschluss (- Abb. 5.2). Die Geschäftslogik für die Verarbeitung der Meldedaten, d.h. alles Fachliche verbleibt dabei vollumfänglich in den Einwohnerkontrollsystemen der Gemeinden. Die Gemeinden haben ausserdem die Möglichkeit, bestimmte Funktionalitäten des über die Plattform gesteuerten Meldeprozesses zu konfigurieren, wie etwa die Downloadmöglichkeit von Dokumenten, um über die Zuzugsgemeinde zu informieren.

Eine wesentliche Voraussetzung für die Orchestrierung des Meldeprozesses bildet die eingangs beschriebene, bereits bestehende Datenaustauschplattform sedex. Im Zusammenspiel von kantonaler Plattform, Einwohnerkontrollsystemen und weiteren relevanten Datenquellen 


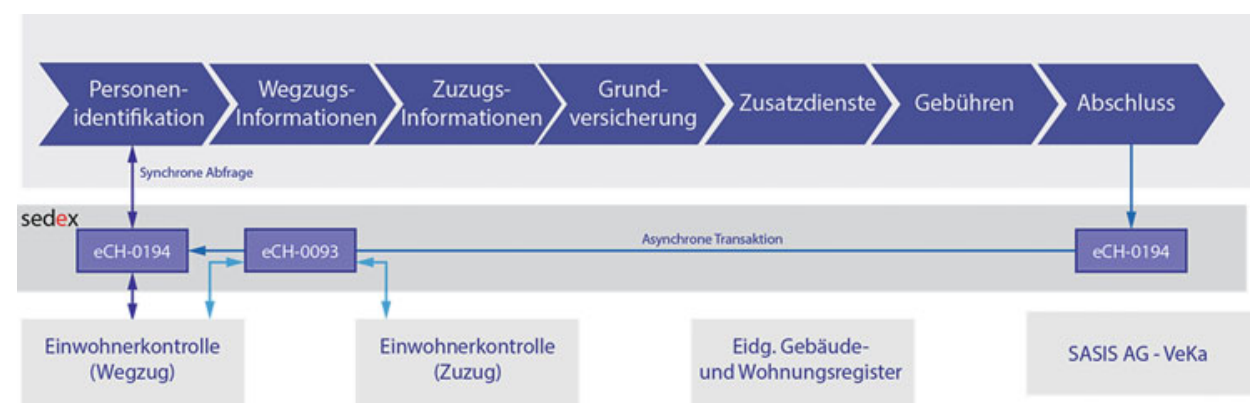

- Abb.5.2 eUmzugZH - Prozess und Lösungselemente

stellt sedex die asynchrone und synchrone Kommunikation sicher. Der Datenausaustausch wird dabei durch verschiedene $\mathrm{CH}$-Standards für das elektronische Meldewesen geregelt. eCH 0194 ist ein eigens für den elektronischen Umzug geschaffener Standard, der die Meldungen zwischen Umzugsplattform und EK-Systemen der Gemeinden definiert (eCH 2015b). Er ermöglicht die synchrone Identifikation einer umziehenden Person zu Beginn des Prozesses mittels Webservices und regelt in der Folge auch alle asynchronen Datenflüsse zwischen der kantonalen Plattform und den EK-Systemen. Ist ein EK-System nicht an sedex angeschlossen, kann der Nutzer die Daten manuell erfassen. Für den Datenaustausch zwischen den EKSystemen kommt der bereits bestehende Standard eCH-0093 zum Einsatz (eCH 2015a). Über Webservices angebunden sind ausserdem das eidgenössische GWR (Gebäude- und Wohnregister), mit dem die neue Wohnadresse geprüft wird und das VeKa-Center, das die OnlinePrüfung der Krankenversicherungsnummer prüft.

\section{- Organisatorische Voraussetzungen}

Sowohl bei der Konzeption und Umsetzung der beschriebenen Lösung als auch für den Betrieb von eUmzugZH spielt die enge Kooperation von Kanton und Gemeinden eine Schlüsselrolle. Zu Beginn des Projekts 2014 wurde daher eine Projektorganisation mit Vertretern von Kanton und Gemeinden gebildet, in die auch die Anbieter der Einwohnerkontrollsysteme einbezogen wurden, um die nahtlose Umsetzung sicherzustellen (• Abb. 5.3).

Die für die Kooperation von Kanton und Gemeinden in E-Government-Projekten zuständige Organisation egovpartner.zh.ch nahm ihre Rolle während der Realisierung im Projektausschuss wahr. Sie vertritt den Kanton Zürich ausserdem im nationalen Projekt A1.12 eUmzug$\mathrm{CH}$. Die Projektorganisation stellte die Realisierung, Pilotierung und Einführung von eUmzug in folgenden Phasen sicher:

1. Projektidee und Machbarkeitsstudie (2014, abgeschlossen)

2. Konzeptphase (2015, abgeschlossen)

3. Implementierungsphase (2015, abgeschlossen)

4. Abnahmetests (November bis März 2015, abgeschlossen)

5. Freigabe durch den Projektausschuss (März 2016)

6. Einführung bei den Gemeinden:

- Phase 1: Pilotierung mit acht Gemeinden (April 2016, abgeschlossen)

- Phase 2: Einführung bei weiteren Gemeinden (rund 30, Stand Juni 2016)

- Phase 3: Flächendeckende Ausdehnung im Kanton Zürich (Juli bis Dezember 2016) 


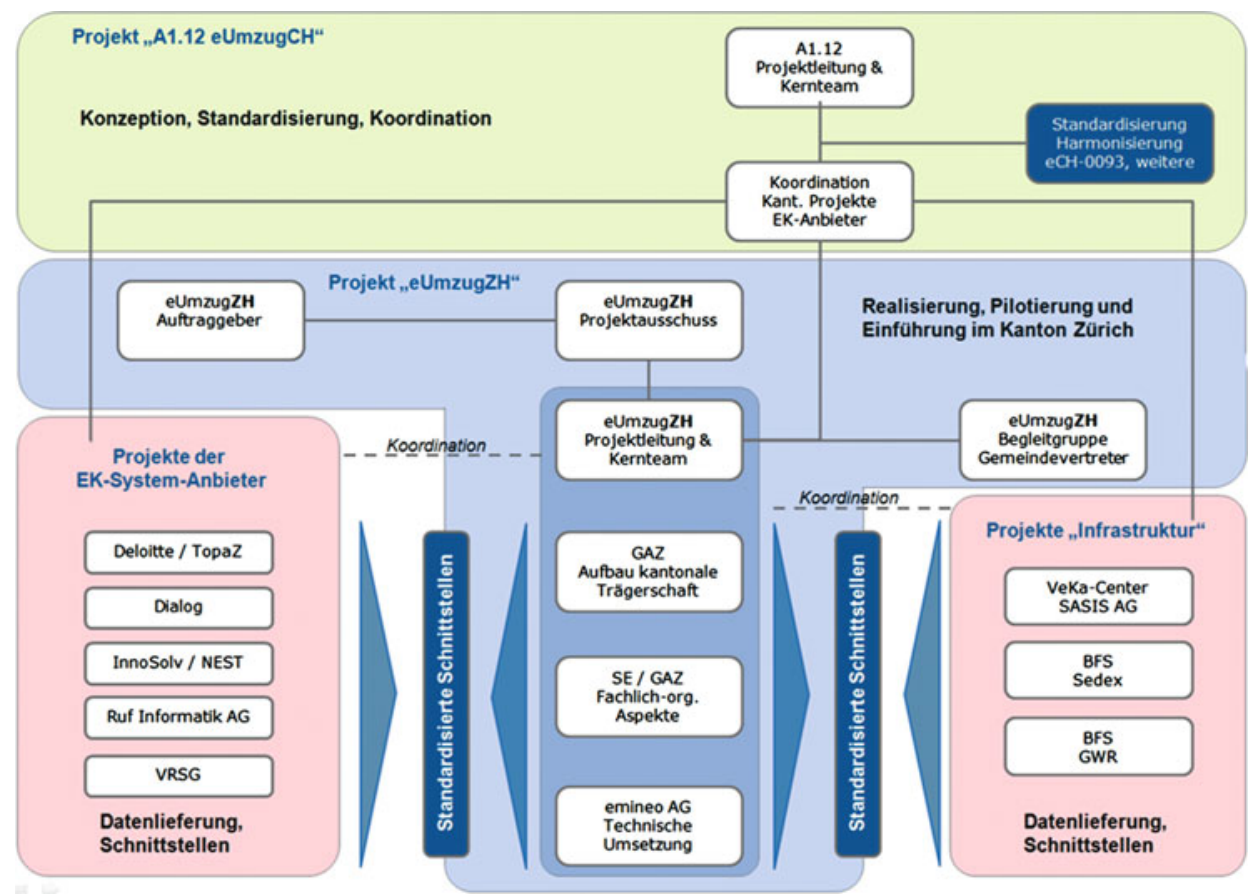

- Abb.5.3 Projektorganisation für die Umsetzung von eUmzugZH im Kontext eUmzugCH

Bei der Umsetzung wurde ein besonderer Schwerpunkt auf das Testen der Lösung in Zusammenarbeit mit den Pilotgemeinden gelegt. Ein nicht funktionaler Prototyp erlaubt frühzeitig, Inputs zur Optimierung der Benutzerführung einzuholen. In umfangreichen Abnahmetests, in denen Gemeindevertreter und Realisierungspartner in unterschiedliche Rolle schlüpften, wurde die Benutzerfreundlichkeit des Systems geprüft und verbessert. Im Fokus stand die intuitive Nutzung des Systems durch die Einwohner und Einwohnerinnen, um einen Betrieb ohne Supportorganisation möglich zu machen.

I) Testen, testen, testen. Wir haben in einem nie dagewesenen Ausmass getestet, um sicherzustellen, dass das System intuitiv und ohne Supportorganisation eingesetzt werden kann. Die positiven Feedbacks von Nutzern aus der Bevölkerung bestätigen die Wirksamkeit dieser Massnahmen (Lukas Steudler, Leiter Geschäftsstelle egovpartner.zh.ch).

Den Betrieb, d. h. die fachliche Trägerschaft für eUmzugZH, übernimmt nach der Einführung das Gemeindeamt des Kantons Zürich, das bereits als organisatorische und finanzielle Verbindungsstelle zwischen Kanton und Gemeinden agiert.

\section{- Rechtliche Voraussetzungen}

Um der zunehmenden Relevanz des Meldewesens Rechnung zu tragen, wurden die Bestimmungen des Melde- und Einwohnerregisterwesens im Kanton Zürich grundlegend überarbeitet, von der Revision des Gemeindegesetztes entkoppelt und in einem eigenen Gesetz geregelt. Seit 1. Januar 2016 ist das MERG $^{4}$ (Gesetz über das Meldewesen und die Einwohnerregister) in

\footnotetext{
${ }^{4}$ Gesetz über das Meldewesen und die Einwohnerregister (MERG), In Kraft seit 01.01.2016, ZH-Lex 142.1.
} 
Kraft und regelt für Kanton und Gemeinden verschiedene Aspekte der Datenerhebung und Datenhaltung sowie des Datenaustauschs. MERG (Art. 15) verpflichtet die Gemeinden im Kanton Zürich explizit, den Meldepflichtigen eine elektronische Umzugsmeldung anzubieten. Diese neue Rechtsgrundlage erhöht den Druck auf die Gemeinden, nach Ablauf der Übergangsfrist an der flächendeckenden Umsetzung von eUmzugZH mitzuwirken.

\section{- Status und Wirkung}

Zwei Monate nach Einführung wird eUmzugZH bereits rege von der Bevölkerung genutzt. Rund 900 Umzüge wurden in dieser Zeit über die Plattform gemeldet. Nutzer benötigen im Schnitt 15 min, um den Prozess vollständig zu durchlaufen. Die Reaktionen, die über ein Feedbackformular am Ende des Prozesses eigefangen werden, sind sehr positiv. Das umfangreiche Testen der Lösung zahlt sich durch eine problemfreie Nutzung und eine hohe Kundenzufriedenheit aus.

Die flächendeckende Ausdehnung schreitet weiter fort. Stand Juli 2016 bieten 49 Gemeinden eUmzugZH an. 153 der 168 Zürcher Gemeinden haben ihre Teilnahme an eUmzugZH zugesagt. Damit stünde der elektronische Umzug rund 1,3 der 1,4 Mio. Einwohner des Kantons Zürich zur Verfügung. Dies entspricht 1/8 der Schweizer Bevölkerung.

Auch der Transfer der Ergebnisse von eUmzug $\mathrm{ZH}$ in das nationale Projekt eUmzugCH hat bereits stattgefunden. Ein Referenzmodell eUmzugCH wurde erarbeitet, das die Prozessmodelle, Datenobjekte, Schnittstellen und verwendete Standards dokumentiert (ISB 2015b). Es kann für Umsetzungsprojekte in anderen Kantonen angewandt werden. Auch in Leitfaden für die Einführung in Gemeinden wird erarbeitet. Ein Verbund eUmzug mit anderen Kantonen für den Erfahrungsaustausch und die gemeinsame Weiterentwicklung des Referenzmodells ist geplant.

\subsection{Fazit}

Mit eUmzugZH hat der Kanton Zürich in der Schweiz eine Vorreiterrolle übernommen. Der Ansatz, die Umsetzung und rasche flächendeckende Ausdehnung über eine kantonale Plattform sicherzustellen, war erfolgreich. Damit verbunden war die anspruchsvolle Koordination der Interessen von Bund, Kantonen, Gemeinden und Anbietern der Einwohnerkontrollsysteme. Ergebnis ist eine von der Bevölkerung bereits nach kurzer Zeit akzeptierte E-GovernmentDienstleistung mit spürbarem Nutzen.

Die Fallstudie macht deutlich, dass sich auch die öffentliche Hand den Herausforderungen und Chancen des digitalen Wandels stellt. Dabei sind innovative und flexible Ansätze gefragt, um die Struktur- und Systemgrenzen im föderativen System der Schweiz zu überwinden. Gelingt dies, wie im Fall von eUmzugZH, können nachhaltige Veränderungen in der Kooperation von Bund, Kanton und Gemeinden, in den Prozessen und insbesondere für die Bevölkerung stattfinden. - Abb. 5.4 fasst diese Auswirkungen im Kontext des Studienframeworks zusammen. 


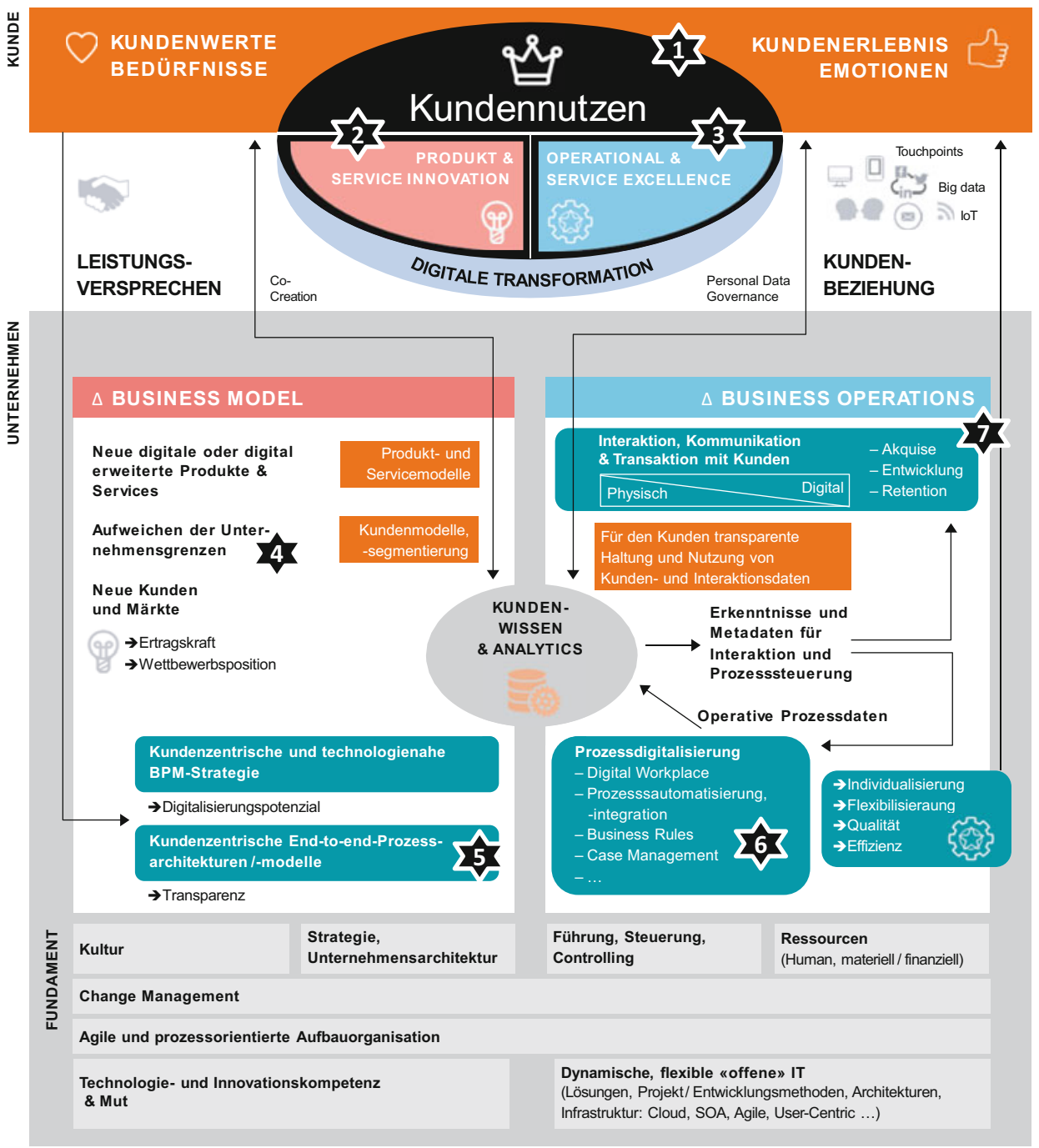

- Abb.5.4 eUmzugZH Fallstudie - Kernaspekte im Kontext des Studienframeworks

\section{Mit welcher Zielsetzung und mit welcher Wirkung wird digital transformiert? Welcher Kun-} dennutzen wird angestrebt bzw. wurde bereits realisiert?

\section{Kundennutzen: einfache elektronische Umzugsmeldung in einem Schritt}

Die Zielsetzung, aus der Perspektive der umziehenden Einwohner und Einwohnerinnen einen durchgängigen, einfach verständlichen und transparenten Meldeprozess anzubieten wurde mit eUmzugZH realisiert. Dies zeigt das positive Feedback der Bevölkerung nach den ersten rund 900 elektronisch abgewickelten Umzugsmeldungen. Das geschaffene Referenzmodell und der Erfahrungsaustausch zwischen den Kantonen schaffen die Grundlagen, damit dieser E-Government-Dienst in absehbarer Zukunft auch kantonsübergreifend der Schweizer Bevölkerung angeboten werden kann. 


\section{Produkt- und Service-Innovation: Kanton Zürich als Vorreiter für eUmzugCH \\ Mit eUmzugZH ist der Kanton Zürich der erste Kanton in der Schweiz, der elektronische Umzugsmeldungen flächendeckend anbietet. Er hat damit die Vorreiterrolle in der Schweiz eingenommen und positive Signale für die Umsetzung weiterer E-Government-Dienste gesetzt.}

\section{Operational \& Service Excellence: medienbruchfreie Umzugsmeldungen \\ eUmzugZH bringt nicht nur Vorteile für die Bevölkerung, sondern reduziert auch Aufwände auf Seiten der Gemeinden. Umzugsmeldungen gehen ohne Medienbrüche, einheitlich und mit hoher Datenqualität bei den Gemeinden ein und können an einem Ort abgearbeitet werden. Die Anzahl der Meldungen am Schalter werden sukzessive zurückgehen, so dass mehr Zeit für komplexere Beratungen der Einwohner und Einwohnerinnen bleibt.}

\section{Was wurde bzw. wird digital transformiert?}

\section{Business Model: kantonale Plattform als Umsetzungshebel}

Plattformen, die Partner vernetzen, um Produkte und Dienstleistungen zu erbringen, sind ein wesentliches Element der digitalen Transformation. Mit eUmzugZH hat der Kanton gemeinsam mit den Gemeinden eine solche Plattform geschaffen. Realisiert, finanziert und betrieben vom Kanton, versetzt die Plattform Gemeinden in die Lage, ihren Einwohnern elektronische Umzugsmeldungen rasch und über die Gemeindegrenzen hinaus anzubieten. Die Hoheit der Gemeinden über das Meldewesen und die Einwohnerregister in der föderalen Schweiz bleibt dabei unangetastet. Dieses Grundprinzip ist auch im Referenzmodell eUmzugCH abgebildet (ISB 2015b). Auch die Interessen der privatwirtschaftlichen Anbieter der Einwohnerkontrollsysteme, die ihren Beitrag zur Innovation des Meldewesens leisten, bleiben gewahrt. Potenzial besteht in der Einbindung von weiteren Datenbezügern, wie z. B. der Schweizerischen Post oder Telekommunikationsanbietern, die ein Interesse an digitalisierten Umzugsmeldungen haben. Die Plattform bietet hierfür alle Voraussetzungen. Im Sinne einer raschen Umsetzung wurde darauf jedoch in einem ersten Schritt verzichtet.

\section{End-to-end Prozessarchitekturen: wiederverwendbare Referenzprozesse}

Im Rahmen der Konzeption auf nationaler und kantonaler Ebene entstanden detaillierte Prozessmodelle, die als Grundlage für die Umsetzung dienten und Eingang in eCH-Standards und das Referenzmodell eUmzugCH fanden. Damit wurde die fachliche Grundlage für die Durchgängigkeit des Prozesses unabhängig von den eingesetzten Technologien geschaffen.

\section{Wie bzw. womit wurde transformiert?}

\section{6rozessdigitalisierung: ZHservices als Transaktionsplattform}

Die kantonale Transaktionsplattform ZHservices in Kombination mit den eCH-Standards und sedex, der nationalen Infrastruktur für den Datenaustausch, stellen die durchgängige Digitalisierung der Meldeprozesse und die einheitliche Benutzeroberfläche für die Bevölkerung sicher.

\section{Interaktion mit der Bevölkerung: digital und physisch}

Auch wenn die Einfachheit der Online-Lösung überzeugt, bleibt die persönliche Interaktion am Schalter weiterhin möglich. Auf diese Weise ist sichergestellt, dass die Bedürfnisse aller Bevölkerungsgruppen erfüllt werden können. 


\section{Literatur}

BfS. (ohne Jahr). sedex. Von Bundesamt für Statistik: http://www.bfs.admin.ch/bfs/portal/de/index/news/00/00/02. html. Abgerufen am 13.6.2016.

eCH (2015a). eCH-0093: Prozess Wegzug Zuzug. http://www.ech.ch/vechweb/page?p=dossier\& documentNumber $=\mathrm{eCH}-0093 \&$ documentVersion=2.0. Abgerufen am 13.6.2016.

eCH (2015b). eCH-0194: Schnittstellenstandard eUmzug. http://www.ech.ch/vechweb/page?p=dossier\& documentNumber=eCH-0194. Abgerufen am 13.6.2016.

eCH. (ohne Jahr). E-Government Standards. Von http://www.ech.ch/. Abgerufen am 13.6.2016.

eGovernmentZH (2012). Vereinbarung zur Zusammenarbeit zwischen dem Kanton Zürich und den Gemeinden im Bereich E-Government. http://www.egovpartner.zh.ch/internet/microsites/egovpartner/de/ueber_uns.html. Abgerufen am 13.6.2016.

egovpartner.zh.ch. (ohne Jahr). eUmzugZH. Von http://www.egovpartner.zh.ch/internet/microsites/egovpartner/ de/projekte/p006_eumzugzh.html. Abgerufen am 13.6.2016.

ISB (2015a). Informatiksteuerungsorgan des Bundes, Geschäftsstelle E-Government Schweiz: Abschlusspublikation E-Government Schweiz 2008-2015. Bern. https://www.egovernment.ch/index.php/download_file/force/332/ 3336/. Abgerufen am 13.6.2016.

ISB (2015b). Informatiksteuerorgan des Bundes: Referenzmodell eUmzugCH. https://www.egovernment.ch/de/ umsetzung/schwerpunktplan/e-umzug-schweiz/. Abgerufen am 13.6.2016.

ISB (2016). Informatiksteuerungsorgan des Bundes, Geschäftsstelle E-Government Schweiz: E-Government Strategie Schweiz. https://www.egovernment.ch/de/umsetzung/e-government-strategie/. Abgerufen am 13.6.2016.

VSED (2012). Verband Schweizerischer Einwohnerdienste: Fachkonzept A1.12 Elektronische Meldung und Abwicklung Adressänderung, Wegzug, Zuzug.

Open Access Dieses Kapitel wird unter der Creative Commons Namensnennung 4.0 International Lizenz (http://creativecommons.org/licenses/by/4.0/deed.de) veröffentlicht, welche die Nutzung, Vervielfältigung, Bearbeitung, Verbreitung und Wiedergabe in jeglichem Medium und Format erlaubt, sofern Sie den/die ursprünglichen Autor(en) und die Quelle ordnungsgemäß nennen, einen Link zur Creative Commons Lizenz beifügen und angeben, ob Änderungen vorgenommen wurden.

Die in diesem Kapitel enthaltenen Bilder und sonstiges Drittmaterial unterliegen ebenfalls der genannten Creative Commons Lizenz, sofern sich aus der Abbildungslegende nichts anderes ergibt. Sofern das betreffende Material nicht unter der genannten Creative Commons Lizenz steht und die betreffende Handlung nicht nach gesetzlichen Vorschriften erlaubt ist, ist für die oben aufgeführten Weiterverwendungen des Materials die Einwilligung des jeweiligen Rechteinhabers einzuholen.

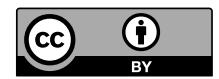

